\title{
Long-time behaviour of solutions to a singular heat equation with an application to hydrodynamics
}

\author{
Georgy Kitavtsev* and Roman M. Taranets ${ }^{\dagger}$
}

March 25, 2022

\begin{abstract}
In this paper, we extend the results of [1] by proving exponential asymptotic $H^{1}$ convergence of solutions to a one-dimensional singular heat equation with $L^{2}$-source term that describe evolution of viscous thin liquid sheets while considered in the Lagrange coordinates. Furthermore, we extend this asymptotic convergence result to the case of a time inhomogeneous source. This study has also independent interest for the porous medium equation theory.
\end{abstract}

\section{Introduction}

In this paper, we study the long-time behaviour of solutions to the initial boundary value problem for a singular heat equation in the presence of an external source term:

$$
\begin{gathered}
u_{t}=\nu\left(u^{-2} u_{x}\right)_{x}+f(x, t) \text { in } Q_{T}:=(0,1) \times(0, T), \\
u_{x}(0, t)=u_{x}(1, t)=0 \text { for } t \in(0, T), \\
u(x, 0)=u_{0}(x) \text { for } x \in(0,1),
\end{gathered}
$$

with $T>0$ and $\nu>0$. Additionally, we assume zero mean of the external force:

$$
\int_{0}^{1} f(x, t) d x=0 \quad \text { for all } t \in(0, T) .
$$

Let us point out that equation (1) without source term $f(x, t)$ was considered first in [2] and is related to the porous-media equations [3]:

$$
u_{t}=\Delta u^{m}, \quad m \geqslant-1 .
$$

*Mathematical Institute, University of Oxford, Woodstock Road, Oxford OX2 6GG, UK

$\dagger$ Institute of Applied Mathematics and Mechanics of the NASU, Dobrovolskogo Str. 1, 84100, Sloviansk, Ukraine 
Indeed, for the critical exponent $m=-1$ the latter equation coincides with (11) after the reversion of the time variable. There exists also a relation between (11) and the classical heat equation through the Lie-Bäcklund transform investigated in [4 6] that allows to find special exact solutions to the former. We do not pursue this approach in this study. Let us also mention recent study [7] of solutions to (11) with a time dependent source term $f(x, t)$ and an application to the Penrose-Fife phase field system [8, 9], in which (1) similar to the original study [2] appears as an equation for temperature distribution.

By contrast, the motivation of this study for considering problem (11)-(4) comes from the recent connection found in [1] of it to the degenerate parabolic system

$$
\begin{aligned}
v_{t}+v v_{y} & =\frac{\nu}{h}\left[h v_{y}\right]_{y}, \\
h_{t} & =-(h v)_{y},
\end{aligned}
$$

describing evolution of the free surface $h(y, t)$ and averaged lateral velocity $v(y, t)$ of a $2 \mathrm{D}$ viscous thin sheet in the absence of surface tension with $(y, t) \in Q_{T}$ and considered with the following boundary conditions:

$$
h_{y}(0, t)=h_{y}(1, t)=v(0, t)=v(1, t)=0 \quad \text { for } \quad t \in(0, T),
$$

and initial data having positive height:

$$
h(y, 0)=h_{0}(y)>0, v(y, 0)=v_{0}(y) \quad \text { for } \quad y \in(0,1) .
$$

We note that system (6) is also a special example of the viscous Saint-Venant or shallowwater equations that were derived and studied extensively in the last three decades, see [1014] and references therein.

It was shown in [1, section 3] that system (6) and equation (10) considered with the special time-independent source term:

$$
f(x, t)=f_{0}(x):=\frac{M}{h_{0}(y(x, 0))}\left[v_{0}(y(x, 0))+\nu \frac{h_{0, y}(y(x, 0))}{h_{0}(y(x, 0))}\right]_{y}
$$

are related by the Eulerian-to-Lagrangian coordinate transformation:

$$
y_{x}(x, t)=\frac{M}{h(y(x, t), t)}, \quad y_{t}(s, t)=v(y(x, t), t), \quad y(x, 0)=M \int_{0}^{x} \frac{d s}{h_{0}(s)}
$$

with constant $M>0$ denoting the total conserved mass of the viscous sheet:

$$
M(t):=\int_{0}^{1} h(y, t) d y=M \quad \text { for all } t \in(0, T) .
$$

Additionally, one defines function

$$
u(x, t):=\frac{M}{h(y(x, t), t)} .
$$


The conservation of mass (10) is ensured by boundary conditions (17) and pertains according to transformation (9) also for the corresponding solutions to problem (11)-(4) with (8) in the following form:

$$
\int_{0}^{1} u(x, t) d x=1 \quad \text { for all } t \in(0, T) .
$$

In [1, Theorem 4.1] the authors have shown that without the source term $f(x, t) \equiv 0$ solutions to (11)-(41) asymptotically decay to the constant profile $u_{\infty}=1$ in $H^{1}$-norm. Besides, basing on numerical simulations of system (6), it was conjectured in [1] (see Remark 4.3 and Fig. 2 there) that in the case of time-independent source $f(x, t)=f_{0}(x)$ solutions to (1)-(4) converge to a unique non-constant positive steady state $u_{\infty}$ solving the stationary version of (1)-(3), namely,

$$
u_{\infty}(x)=\frac{\nu}{\int_{0}^{x} \int_{0}^{y} f(s) d s d y+C_{\nu}}>0
$$

with $C_{\nu}$ being a unique root, due to (12), of the following equation:

$$
\int_{0}^{1}\left[\int_{0}^{x} \int_{0}^{y} f(s) d s d y+C_{\nu}\right]^{-1} d x=\nu^{-1}
$$

Indeed, for $f \in L^{2}(0,1)$ the double integral in (13)-(14) is $C^{1}[0,1]$ function and, therefore, for any $\nu>0$ positive function $u_{\infty} \in H^{2}(0,1)$ and constant $C_{\nu}$ are uniquely defined.

Moreover, numerical simulations of [1] indicated that the corresponding solutions to the shallow-water system (6) defined through transformation (9) converge as time goes to infinity to the corresponding limit profiles:

$$
y_{\infty}(x)=\int_{0}^{x} u_{\infty}(s) d s, \quad h_{\infty}\left(y_{\infty}(x)\right)=\frac{M}{u_{\infty}(x)}, \quad v_{\infty}\left(y_{\infty}(x)\right)=0 \quad \text { for } x \in(0,1),
$$

which are defined explicitly by the initial data $\left(h_{0}, v_{0}\right)$ as, due to (8), one has

$$
u_{\infty}(x)=\frac{\nu}{\int_{0}^{x} v_{0}\left((y(s, 0)) d s+\frac{\nu}{M}\left(h_{0}\left(y(x, 0)-h_{0}(0)\right)+C_{\nu}\right.\right.} .
$$

This intriguing result implies selection of a single attracting steady-state (15) to system (6) depending only on the given initial data $\left(h_{0}, v_{0}\right)$ through $u_{\infty}$ in (16). We note that this selective long-time asymptotic behaviour can not be easily identified in Eulerian formulation ([6), because the set of the steady states to (6) consists of pairs $\left(h_{\infty}, 0\right)$ with any sufficiently smooth $h_{\infty}(x)$ satisfying boundary conditions in (7). We are not aware if this long-time solution behaviour was shown analytically or numerically for the shallow-water system (6) in the literature before study [1].

In this article, to put this result on a firm basis we prove that solutions to singular heat equation (11)-(3) considered with $L^{2}$-source term satisfying (44) exponentially converge to 
$u_{\infty}$ in $H^{1}$-norm, provided certain integro-algebraic relations between $\nu, f(x, t)$ and $u_{0}$ hold (see conditions (17)-(19) and (41)-(45) in Theorems 2.1 and 3.1, respectively).

The rest of the article is organised as follows. In section 2, we prove an exponential asymptotic decay result for the time homogeneous case $f(x, t)=f_{0}(x)$ (Theorem 2.1 and Corollary 2.2). In section 3 , we generalise it to the time inhomogeneous case (Theorem 3.1 and Corollary 3.3). In this case the rate of the asymptotic convergence to the limit profile is determined by the rate of $L^{2}$-convergence of the right-hand side $f(\cdot, t)$ as time goes to infinity. In Examples 2.4 and 3.3 we calculate the explicit form of the derived asymptotic convergence estimates for two concrete examples of source function $f$. In particular, Example 2.4 shows how to transform these estimates into the corresponding ones for the solutions to viscous shallow-water system (6) -(17) using Eulerian-to-Lagrangian transformation (9).

Our proofs use essentially the energy dissipation methods, see e.g. [15, 16] and references therein. In particular, Theorem 3.1 extends the results of [17] on asymptotic exponential convergence to the self-similar Barenblatt's profiles of solutions to porous-media equation (5) for the case $m \geqslant 1$ considered also with a time-dependent source $f(x, t)$. We also note that, in contrast to system considered in [17], (11)-(4) posses conservation of mass (12) motivated by their relation to system (6) via transformation (9) discussed above.

Finally, we note that establishing a rigorous correspondence between systems (6) and (11) -(41) with special right-hand side (81) would demand analysis of regularity for their weak solutions [18] and is out of the scope of this study.

\section{Time homogeneous case}

In the case of $f(x, t)=f_{0}(x) \in L^{2}(0,1)$ in (1) -(4), we prove the following result.

Theorem 2.1. Let

$$
0<u_{0}(x) \in H^{1}(0,1): R_{0}:=\left\|\left(u_{0}^{-1}\right)_{x}\right\|_{2}<\infty,
$$

and

$$
f_{0}(x) \in L^{2}(0,1): \int_{0}^{1} f_{0}(x) d x=0 \quad \text { and } P_{0}:=\left\|\int_{0}^{x} f_{0}(s) d s\right\|_{2}<\infty .
$$

If, additionally,

$$
R_{0} \in[0,1) \quad \text { and } \quad \nu \in\left(\frac{2 P_{0}}{1-R_{0}},+\infty\right)
$$

then a weak solution $u(x, t)$ to problem (11)-(3) considered with (12) satisfies

$$
0<\frac{\nu}{\nu\left(1+R_{0}\right)+2 P_{0}} \leqslant u(x, t) \leqslant \frac{\nu}{\nu\left(1-R_{0}\right)-2 P_{0}}<\infty \quad \text { for all }(x, t) \in Q_{T} .
$$

Moreover, $u^{-1}(\cdot, t)$ converges to the inverse of steady state solution $u_{\infty}^{-1}$ defined by (13)-(14) considered with $f=f_{0}$, namely,

$$
\left\|u^{-1}(\cdot, t)-u_{\infty}^{-1}\right\|_{H^{1}} \leqslant\left\|u_{0}^{-1}-u_{\infty}^{-1}\right\|_{H^{1}} \exp \left\{-\frac{\pi^{2} t}{\nu}\left[\nu\left(1-R_{0}\right)-2 P_{0}\right]^{2}\right\} \text { for all } t>0 .
$$


Proof of Theorem 2.1. Introducing a new function $q$ by

$$
q(x, t)=\nu^{\frac{1}{2}} u^{-1}(x, t),
$$

we can rewrite problem (1)-(3) in the form

$$
\begin{gathered}
q_{t}=q^{2}\left(q_{x x}-\nu^{-\frac{1}{2}} f_{0}\right) \text { in } Q_{T}, \\
q_{x}(0, t)=q_{x}(1, t)=0 \text { for } t>0, \\
q(x, 0)=q_{0}(x):=\nu^{\frac{1}{2}} u_{0}^{-1}(x) \quad \text { for } x \in(0,1) .
\end{gathered}
$$

Moreover, by (12) we have

$$
\int_{0}^{1} q^{-1} d x=\int_{0}^{1} q_{0}^{-1} d x=\nu^{-\frac{1}{2}}
$$

Multiplying (23) by $-\left(q_{x x}-\nu^{-\frac{1}{2}} f_{0}\right)$, after integration over $(0,1)$, we get

$$
\frac{d}{d t} \mathcal{E}(q(t))+\int_{0}^{1} q^{2}\left(q_{x x}-\nu^{-\frac{1}{2}} f_{0}(x)\right)^{2} d x=0
$$

where the energy functional is

$$
\mathcal{E}(q(t)):=\int_{0}^{1}\left[\frac{1}{2} q_{x}^{2}+\nu^{-\frac{1}{2}} f_{0}(x) q\right] d x .
$$

Integrating (27) in time, one obtains the energy equality:

$$
\mathcal{E}(q(t))+\iint_{Q_{T}} q^{2}\left(q_{x x}-\nu^{-\frac{1}{2}} f_{0}(x)\right)^{2} d x d t=\mathcal{E}\left(q_{0}\right) .
$$

Let us introduce a set

$$
\mathcal{M}_{\nu}:=\left\{q \in H^{1}(0,1): q_{x}(0)=q_{x}(1)=0, \int_{0}^{1} q^{-1} d x=\nu^{-\frac{1}{2}}\right\} .
$$

Let also $q_{\min }(x)$ be a unique solution of the corresponding Euler-Lagrange equation

$$
q_{x x}=\nu^{-\frac{1}{2}} f_{0}(x) \text { with } q_{x}(0)=q_{x}(1)=0,
$$

describing critical points of the functional $\mathcal{E}$ over $\mathcal{M}_{\nu}$. Hence,

$$
q_{\min }(x)=\frac{\nu^{\frac{1}{2}}}{u_{\infty}(x)} \quad \text { with } u_{\infty}(x) \text { defined in (13) }- \text { (14) }
$$

Next, we look for a lower bound for $\mathcal{E}(q(t))$. Using integration by parts, one can estimate

$$
\int_{0}^{1} f_{0}(x) q d x=-\int_{0}^{1} q_{\min , x} q_{x} d x \leqslant\left\|q_{\min , x}\right\|_{2}\left\|q_{x}\right\|_{2} .
$$


Hence, one has

$$
\mathcal{E}(q(t)) \geqslant \frac{1}{2}\left\|q_{x}\right\|_{2}^{2}-\left\|q_{\min , x}\right\|_{2}\left\|q_{x}\right\|_{2} \geqslant-\frac{1}{2}\left\|q_{\min , x}\right\|_{2}=\min _{q \in \mathcal{M}_{\nu}} \mathcal{E}(q) .
$$

We conclude that the lower semi-continuous bounded from below functional $\mathcal{E}(q)$ attains its unique global minimum $q_{\min }(x)$ on $\mathcal{M}_{\nu}$. According to definitions (17)-(18),

$$
P_{0}=\nu^{1 / 2}\left\|q_{\min , x}\right\|_{2} \text { and } R_{0}=\nu^{-1 / 2}\left\|q_{0, x}\right\|_{2} \text {. }
$$

Next, (28) together with Hölder inequality imply

$$
0 \geqslant \frac{1}{2}\left\|q_{x}(\cdot, t)\right\|_{2}^{2}-\nu^{-1 / 2} P_{0}\left\|q_{x}(\cdot, t)\right\|_{2}-\mathcal{E}\left(q_{0}\right),
$$

and, consequently, the following upper bound for solutions to (23) $-(25)$ :

$$
\left\|q_{x}\right\|_{2} \leqslant \nu^{-1 / 2} P_{0}+\sqrt{\nu^{-1} P_{0}^{2}+2 E\left(q_{0}\right)} \leqslant \nu^{-1 / 2} P_{0}+\sqrt{\nu^{-1} P_{0}^{2}+\nu R_{0}^{2}+2 P_{0} R_{0}},
$$

whence

$$
\left\|q_{x}(\cdot, t)\right\|_{2} \leqslant 2 \nu^{-1 / 2} P_{0}+\nu^{1 / 2} R_{0} \quad \text { for all } t \in(0, T) .
$$

By (26) there exists $x_{0} \in(0,1)$ such that $q\left(x_{0}, t\right)=\nu^{\frac{1}{2}}$. Using this and (30) one estimates:

$$
\left|q(x, t)-\nu^{1 / 2}\right| \leqslant\left\|q_{x}(\cdot, t)\right\|_{2} \leqslant 2 \nu^{-1 / 2} P_{0}+\nu^{1 / 2} R_{0}
$$

The last inequality implies the pointwise estimates

$$
\nu^{1 / 2}-2 \nu^{-1 / 2} P_{0}-\nu^{1 / 2} R_{0} \leqslant q(x, t) \leqslant \nu^{1 / 2}+2 \nu^{-1 / 2} P_{0}+\nu^{1 / 2} R_{0}
$$

for all $(x, t) \in Q_{T}$. From (31) it follows that $q(x, t)>0$ if conditions (19) holds. Furthermore, by (22) and (31) conditions (19) imply estimate (20).

Next, let us introduce

$$
w:=q-q_{\min }
$$

Then

$$
\begin{aligned}
& \mathcal{E}\left(q \mid q_{\min }\right):= \mathcal{E}(q(t))-\mathcal{E}\left(q_{\min }\right)=\frac{1}{2} \int_{0}^{1} w_{x}\left(q+q_{\min }\right)_{x} d x+ \\
& \nu^{-\frac{1}{2}} \int_{0}^{1} f_{0}(x) w d x=-\frac{1}{2} \int_{0}^{1} w\left(q+q_{\min }\right)_{x x} d x+\int_{0}^{1} q_{\min , x x} w d x= \\
&-\frac{1}{2} \int_{0}^{1} w w_{x x} d x=\frac{1}{2} \int_{0}^{1} w_{x}^{2} d x .
\end{aligned}
$$

From here we find that

$$
\frac{d}{d t} \mathcal{E}\left(q \mid q_{\min }\right)=-\int_{0}^{1} w_{t} w_{x x} d x=-\int_{0}^{1} q^{2} w_{x x}^{2} d x \leqslant 0 .
$$


By (24) the Poincaré inequality

$$
\int_{0}^{1} w_{x}^{2} d x \leqslant \frac{1}{\pi^{2}} \int_{0}^{1} w_{x x}^{2} d x
$$

holds. Combining it with (20), one arrives at

$$
\frac{\pi^{2}}{\nu}\left[\nu\left(1-R_{0}\right)-2 P_{0}\right]^{2} \mathcal{E}\left(q \mid q_{\min }\right) \leqslant \int_{0}^{1} q^{2} w_{x x}^{2} d x .
$$

Hence, by (33) we deduce that

$$
\frac{d}{d t} \mathcal{E}\left(q \mid q_{\min }\right)+\frac{\pi^{2}}{\nu}\left[\nu\left(1-R_{0}\right)-2 P_{0}\right]^{2} \mathcal{E}\left(q \mid q_{\min }\right) \leqslant 0 .
$$

From (34) it follows that

$$
0 \leqslant \mathcal{E}\left(q \mid q_{\text {min }}\right) \leqslant \mathcal{E}\left(q_{0} \mid q_{\text {min }}\right) \exp \left\{-\frac{\pi^{2}}{\nu}\left[\nu\left(1-R_{0}\right)-2 P_{0}\right]^{2} t\right\} \rightarrow 0 \text { as } t \rightarrow+\infty .
$$

Therefore, we obtain that

$$
q(\cdot, t) \rightarrow q_{\min } \text { strongly in } H^{1}(0,1) \text { as } t \rightarrow+\infty
$$

and, consequently, (21).

Corollary 2.2. Conditions of Theorem 2.1 imply also the direct exponential asymptotic convergence of $u(\cdot, t)$ to $u_{\infty}$ in $H^{1}(0,1)$. Namely, there exists a constant $C$ depending on $\nu, f_{0}(x)$ and $u_{0}$ such that

$$
\left\|u(\cdot, t)-u_{\infty}\right\|_{H^{1}} \leqslant C\left\|u_{0}-u_{\infty}\right\|_{H^{1}} \exp \left\{-\frac{\pi^{2} t}{\nu}\left[\nu\left(1-R_{0}\right)-2 P_{0}\right]^{2}\right\} \text { for all } t>0 .
$$

Proof. The statement follows from (21), uniform bounds (20) and the following pointwise bounds:

$$
\left(1+\frac{P_{0}}{\nu}\right)^{-1} \leqslant u_{\infty}(x) \leqslant\left(1-\frac{P_{0}}{\nu}\right)^{-1},\left(1+R_{0}\right)^{-1} \leqslant u_{0}(x) \leqslant\left(1-R_{0}\right)^{-1} \text { for } x \in(0,1) .
$$

In the last estimates we used that $R_{0}<1$ and $P_{0}<\nu$ holding by assumption (19).

Example 2.3. Here, we show how the asymptotic convergence result from Theorem 2.1 can be applied to show the corresponding convergence (at least formally) of solutions to the viscous shallow water system (66)-(7). The following example is similar to one presented in [1, Remark 4.3 and Fig. 2] and demonstrates that a solution to (66) starting from a constant $h_{0}(y)=1$ but having non-zero initial velocity $v_{0}(y)$ asymptotically, as time goes to infinity, converges to an inhomogeneous limit profile $h_{\infty}(y)$. 
Firstly, note that Eulerian-to-Lagrangian transformation (9) together with relation (11) allows to rewrite the asymptotic convergence estimate (21) of Theorem 2.1 as one for solution $h(y, t)$ to (6) $-(7)$ in the form:

$\left\|h(y(\cdot, t), t)-h_{\infty}\left(y_{\infty}(\cdot)\right)\right\|_{H^{1}} \leqslant\left\|h_{0}(y(\cdot, 0))-h_{\infty}\left(y_{\infty}(\cdot)\right)\right\|_{H^{1}} \exp \left\{-\frac{\pi^{2} t}{\nu}\left[\nu\left(1-R_{0}\right)-2 P_{0}\right]^{2}\right\}$,

holding for all $t>0$, where

$$
h_{\infty}\left(y_{\infty}(x)\right)=\frac{M}{u_{\infty}(x)}, y_{\infty}(x)=\int_{0}^{x} u_{\infty}(x) d x, \quad \text { and } \quad h_{0}(y(x, 0))=\frac{M}{u_{0}(x)}
$$

are the limit and initial height profiles, respectively. Moreover, by definitions (17)-(18) and again relation (11) one finds that

$$
R_{0}=\frac{1}{M}\left\|h_{0, x}\right\|_{2} \quad \text { and } P_{0}=\frac{\nu}{M}\left\|h_{\infty, x}\right\|_{2} .
$$

Using these, one can write out the right-hand side of (35) solely using $h_{\infty}$ and $h_{0}$ functions as

$$
\begin{aligned}
& \left\|h(y(\cdot, t), t)-h_{\infty}\left(y_{\infty}(\cdot)\right)\right\|_{H^{1}} \\
& \quad \leqslant\left\|h_{0}(y(\cdot, 0))-h_{\infty}\left(y_{\infty}(\cdot)\right)\right\|_{H^{1}} \exp \left\{-\pi^{2} t\left[1-\frac{\left\|h_{0, x}\right\|_{2}+2\left\|h_{\infty, x}\right\|_{2}}{M}\right]^{2}\right\} \text { for all } t>0 .
\end{aligned}
$$

Interestingly, the exponential asymptotic decay rate of (36) turns out to be independent of viscosity $\nu$ in this case.

Next, let us check conditions of Theorem 2.1 and calculate all entries of (36) for the following concrete example of the initial data to (6) :

$$
\begin{aligned}
& h_{0}(y)=h_{0}(y(x, 0))=u_{0}(x)=1, \\
& v_{0}(y)=\frac{1}{2} \sin (\pi y), v_{0}(y(x, 0))=\frac{1}{2} \sin (\pi x), \\
& M=\nu=1
\end{aligned}
$$

having a constant initial height profile.

In this case, according to definitions (8) and (11)

$$
f_{0}(x)=\frac{M}{h_{0}(y(x, 0))}\left[v_{0}(y(x, 0))\right]_{y}=\left[v_{0}(y(x, 0))\right]_{x}=\frac{\pi}{2} \cos (\pi x),
$$

where we again used transformation of variables (9). Hence, by definitions (17)-(18) one calculates:

$$
R_{0}=0 \quad \text { and } P_{0}=\left\|\int_{0}^{x} f_{0}(s) d s\right\|_{2}=\left\|v_{0}(y(x, 0))\right\|_{2}=\frac{1}{2 \sqrt{2}} .
$$

Therefore, in this case

$$
\nu\left(1-R_{0}\right)-2 P_{0}=1-\frac{1}{\sqrt{2}}>0
$$


and all conditions of Theorem 2.1 are satisfied. From equation (1) and definition (11) one obtains

$$
h_{\infty}\left(y_{\infty}(x)\right)-h_{\infty}(0)=\int_{0}^{x} v_{0}(y(s, 0)) d s=\frac{1}{2 \pi}(1-\cos (\pi x)) .
$$

In turn, constant $h_{\infty}(0)$ is determined by the analogue of condition (14):

$$
\int_{0}^{1} \frac{d x}{h_{\infty}\left(y_{\infty}(x)\right)}=1
$$

so that one obtains

$$
h_{\infty}\left(y_{\infty}(x)\right)=\frac{1}{2 \pi}\left(C_{\infty}-\cos (\pi x)\right), \text { where } C_{\infty} \approx 6.37 .
$$

One can also calculate explicitly

$$
\left\|h_{\infty}\left(y_{\infty}(\cdot)\right)-h_{0}(y(\cdot, 0))\right\|_{H^{1}}=\left\|\frac{1}{2 \pi}\left(C_{\infty}-\cos (\pi x)\right)-1\right\|_{H^{1}} \approx 0.37 .
$$

Finally, substituting (38)-(40) into (35) one obtains an explicit asymptotic decay estimate for the solution to (6) with initial data given by (37):

$$
\begin{aligned}
\left\|h(y(\cdot, t), t)-\frac{1}{2 \pi}\left(C_{\infty}-\cos (\pi \cdot)\right)\right\|_{H^{1}} & \leqslant\left\|\frac{1}{2 \pi}\left(C_{\infty}-\cos (\pi x)\right)-1\right\|_{H^{1}} \exp \left\{-\pi^{2} t\left[1-\frac{1}{\sqrt{2}}\right]^{2}\right\} \\
& \leqslant 0.37 \exp \{-0.84 t\} \text { for all } t>0,
\end{aligned}
$$

We note that the last estimate implies also an asymptotic convergence result of $h(\cdot, t)$ to $h_{\infty}$ in original Eulerian coordinates $(y, t)$ as $t \rightarrow \infty$.

\section{Time inhomogeneous case}

In the case of $f \in C\left(0, T ; L^{2}(0,1)\right)$ for all $T>0$ in (1) -(4), we prove the following result.

Theorem 3.1. Let

$$
0<u_{0}(x) \in H^{1}(0,1): R_{0}:=\left\|\left(u_{0}^{-1}\right)_{x}\right\|_{2}<\infty .
$$

Let $f \in C\left(0, T ; L^{2}(0,1)\right)$ for all $T>0$ and $f_{0}, f_{\infty} \in L^{2}(0,1)$ be such that

$$
\left\|f(\cdot, t)-f_{\infty}\right\|_{2} \rightarrow 0 \text { as } t \rightarrow \infty \text { and }\left\|f(\cdot, t)-f_{0}\right\|_{2} \rightarrow 0 \text { as } t \rightarrow 0 .
$$

Assume that

$$
P_{0}:=\left\|\int_{0}^{x} f_{0}(s) d s\right\|_{2}<\infty, \quad N_{\infty}:=\int_{0}^{\infty}\left\|\int_{0}^{x} f_{t}(s, t) d s\right\|_{2} d t<\infty .
$$

If conditions

$$
R_{0} \in[0,1) \text { and } \nu \in\left(\nu_{+},+\infty\right)
$$


hold with

$$
\nu_{+}:=\frac{2 N_{\infty}+\left(1+R_{0}\right) P_{0}+\sqrt{\left(2 N_{\infty}+P_{0}\left(1+R_{0}\right)\right)^{2}-N_{\infty}^{2}\left(1-R_{0}^{2}\right)}}{1-R_{0}^{2}},
$$

then a weak solution $u(x, t)$ to (1)-(3) considered with (12) obeys the uniform bounds

$$
0<A_{-} \leqslant u(x, t) \leqslant A_{+}<\infty
$$

with

$$
A_{ \pm}:=\frac{\nu}{\nu \mp\left[2 N_{\infty}+P_{0}+\sqrt{\left(N_{\infty}+P_{0}\right)^{2}+2\left(N_{\infty}+P_{0}\right) N_{\infty}+\nu^{2} R_{0}^{2}+2 \nu P_{0} R_{0}}\right]}
$$

for all $x \in(0,1)$ and $t>0$.

Furthermore, there exists a unique solution $u_{\infty} \in H^{2}(0,1)$ of the limit equation

$$
-\left(u_{\infty}^{-2} u_{\infty, x}\right)_{x}=\nu^{-1} f_{\infty}(x) \text { with } u_{\infty, x}(0)=u_{\infty, x}(1)=0,
$$

given by (13)-(14) considered with $f=f_{\infty}$, such that $u^{-1}(\cdot, t)$ converges to $u_{\infty}^{-1}$, namely, for all $t>0$ it holds

$$
\left\|u^{-1}(\cdot, t)-u_{\infty}^{-1}\right\|_{H^{1}} \leqslant e^{-B t}\left[\left\|u_{0}^{-1}-u_{\infty}^{-1}\right\|_{H^{1}}+C \int_{0}^{t}\left\|f(., s)-f_{\infty}\right\|_{2}^{2} e^{B s} d s\right] \rightarrow 0,
$$

as $t \rightarrow \infty$, where constants

$$
C:=\frac{1}{\nu^{1 / 2} A_{-}^{2}}+\frac{1}{\nu^{1 / 2} A_{+}^{2}}, \quad B:=\frac{\nu \pi^{2}}{2 A_{+}^{2}} .
$$

Proof of Theorem 3.1. Similar to the proof of Theorem 2.1 by introducing a new function $q$ as

$$
q(x, t)=\nu^{\frac{1}{2}} u^{-1}(x, t),
$$

we can rewrite problem (1)-(3) in the form

$$
\begin{gathered}
q_{t}=q^{2}\left(q_{x x}-\nu^{-\frac{1}{2}} f\right) \text { in } Q_{T}, \\
q_{x}(0, t)=q_{x}(1, t)=0 \quad \text { for } t>0, \\
q(x, 0)=q_{0}(x):=\nu^{\frac{1}{2}} u_{0}^{-1}(x) \quad \text { for } x \in(0,1),
\end{gathered}
$$

equipped with

$$
\int_{0}^{1} q^{-1}(\cdot, t) d x=\int_{0}^{1} q_{0}^{-1} d x=\nu^{-\frac{1}{2}} \text { for } t>0 .
$$

Multiplying (52) by $-\left(q_{x x}-\nu^{-\frac{1}{2}} f\right)$, after integration by parts over $Q_{T}$ and using property (4), one obtains the following energy equality:

$$
\mathcal{E}(q(t))+\int_{0}^{t} \int_{0}^{1} q^{2}\left(q_{x x}-\nu^{-\frac{1}{2}} f\right)^{2} d x+\nu^{-1 / 2} \int_{0}^{t} \int_{0}^{1} q_{x}\left[\int_{0}^{x} f_{\tau} d s\right] d x d \tau=\mathcal{E}\left(q_{0}\right),
$$


with the energy functional

$$
\mathcal{E}(q(\cdot, t)):=\int_{0}^{1}\left[\frac{1}{2} q_{x}^{2}+\nu^{-\frac{1}{2}} f q\right] d x
$$

Applying the Hölder inequality in (56) gives

$$
\left\|q_{x}(\cdot, t)\right\|_{2}^{2} \leqslant 2 \nu^{-1 / 2} P(t)\left\|q_{x}(\cdot, t)\right\|_{2}+2 \nu^{-1 / 2} \int_{0}^{t}\left\|q_{x}(\cdot, \tau)\right\|_{2} N^{\prime}(\tau) d \tau+2 \mathcal{E}\left(q_{0}\right),
$$

where we introduced functions

$$
P(t):=\left\|\int_{0}^{x} f(s, t) d s\right\|_{2}, \quad N(t):=\int_{0}^{t}\left\|\int_{0}^{x} f_{\tau}(s, \tau) d s\right\|_{2} d \tau .
$$

Using these, $f \in L^{\infty}\left(0, T ; L^{2}(0,1)\right)$ and (43) one estimates

$$
\begin{aligned}
2 P(t) P^{\prime}(t) & =\frac{d}{d t} P^{2}(t)=\frac{d}{d t} \int_{0}^{1}\left[\int_{0}^{x} f(s, t) d s\right]^{2} d x \\
& =2 \int_{0}^{1}\left[\int_{0}^{x} f(s, t) d s\right]\left[\int_{0}^{x} f_{t}(s, t) d s\right] d x \\
& \leqslant 2 P(t)\left(\int_{0}^{1}\left[\int_{0}^{x} f_{t}(s, t) d s\right]^{2} d x\right)^{1 / 2}=2 P(t) N^{\prime}(t) .
\end{aligned}
$$

Hence, one deduces bounds

$$
P^{\prime}(t) \leqslant N^{\prime}(t) \text { and } P(t) \leqslant N_{\infty}+P_{0}
$$

where we used definitions (43). Using (159), one can rewrite (158) as

$$
\left(\left\|q_{x}(\cdot, t)\right\|_{2}-\nu^{-1 / 2}\left(N_{\infty}+P_{0}\right)\right)^{2} \leqslant \nu^{-1}\left(N_{\infty}+P_{0}\right)^{2}+2 \nu^{-1 / 2} \int_{0}^{t}\left\|q_{x}(\cdot, \tau)\right\|_{2} N^{\prime}(\tau) d \tau+2 \mathcal{E}\left(q_{0}\right) .
$$

By introduction of the new nonnegative function

$$
v(t):=\left(\left\|q_{x}(\cdot, t)\right\|_{2}-\nu^{-1 / 2}\left(N_{\infty}+P_{0}\right)\right)^{2},
$$

the last inequality together with (59) and (57) implies:

$$
\begin{aligned}
v(t) & \leqslant \nu^{-1}\left(N_{\infty}+P_{0}\right)^{2}+2 \nu^{-1 / 2} \int_{0}^{t} v^{1 / 2}(\tau) N^{\prime}(\tau) d \tau+2 \nu^{-1} \int_{0}^{t}\left(N_{\infty}+P_{0}\right) N^{\prime}(\tau) d \tau+2 \mathcal{E}\left(q_{0}\right) \\
& =\nu^{-1}\left(N_{\infty}+P_{0}\right)^{2}+2 \nu^{-1 / 2} \int_{0}^{t} v^{1 / 2} N^{\prime}(\tau) d \tau+2 \nu^{-1}\left(N_{\infty}+P_{0}\right) N(t)+2 \mathcal{E}\left(q_{0}\right) \\
& \leqslant a_{0}+2 \nu^{-1 / 2} \int_{0}^{t} v^{1 / 2}(\tau) N^{\prime}(\tau) d \tau,
\end{aligned}
$$


where

$$
a_{0}:=\nu^{-1}\left(N_{\infty}+P_{0}\right)^{2}+2 \nu^{-1}\left(N_{\infty}+P_{0}\right) N_{\infty}+\nu R_{0}^{2}+2 P_{0} R_{0},
$$

with $R_{0}$ being defined in (41).

The last inequality after an application of Bihari-LaSalle lemma [19] to $v(t)$, while using $a_{0} \geqslant 0$ and $N^{\prime}(t) \geqslant 0$, implies

$$
v^{1 / 2}(t) \leqslant a_{0}^{1 / 2}+\nu^{-1 / 2} N(t) \leqslant a_{0}^{1 / 2}+\nu^{-1 / 2} N_{\infty},
$$

which, in turn, using definition (60) gives

$$
\left\|q_{x}(\cdot, t)\right\|_{2} \leqslant a_{0}^{1 / 2}+2 \nu^{-1 / 2} N_{\infty}+\nu^{-1 / 2} P_{0} .
$$

In turn, this together with (55) implies

$$
\begin{aligned}
\left|q(x, t)-\nu^{1 / 2}\right| & \leqslant \int_{0}^{1}\left|q_{x}(x, t)\right| d x \leqslant\left\|q_{x}(\cdot, t)\right\|_{2} \\
& \leqslant a_{0}^{1 / 2}+2 \nu^{-1 / 2} N_{\infty}+\nu^{-1 / 2} P_{0}
\end{aligned}
$$

and, consequently,

$$
\nu^{1 / 2}-a_{0}^{1 / 2}-2 \nu^{-1 / 2} N_{\infty}-\nu^{-1 / 2} P_{0} \leqslant q(x, t) \leqslant \nu^{1 / 2}+a_{0}^{1 / 2}+2 \nu^{-1 / 2} N_{\infty}+\nu^{-1 / 2} P_{0}
$$

hold for all $x \in(0,1)$ and $t>0$. Estimates (61) together with definition (51) imply the uniform pointwise bounds (46)-(47) provided

$$
\nu-a_{0}^{1 / 2} \nu^{1 / 2}-2 N_{\infty}-P_{0}>0 .
$$

This is equivalent to the following system of inequalities:

$$
\begin{aligned}
\nu & >2 N_{\infty}+P_{0}, \\
1 & >R_{0}^{2}, \\
\left(1-R_{0}^{2}\right) \nu^{2}-2 \nu\left(2 N_{\infty}+P_{0}+R_{0} P_{0}\right)+N_{\infty}^{2} & >0 .
\end{aligned}
$$

By solving the last inequality explicitly this system turns into:

$$
\begin{aligned}
\nu & >2 N_{\infty}+P_{0}, \\
1 & >R_{0}^{2}, \\
\nu & \in\left(0, \nu_{-}\right) \cup\left(\nu_{+},+\infty\right),
\end{aligned}
$$

where

$$
\nu_{ \pm}:=\frac{2 N_{\infty}+\left(1+R_{0}\right) P_{0} \pm \sqrt{\left(2 N_{\infty}+P_{0}\left(1+R_{0}\right)\right)^{2}-N_{\infty}^{2}\left(1-R_{0}^{2}\right)}}{1-R_{0}^{2}}
$$

It is easy to check that $\nu_{-}<2 N_{\infty}+P_{0}$ for all nonnegative $N_{\infty}, P_{0}$ and $R_{0}$. Therefore, the last system of inequalities is equivalent to conditions (44)-(45). 
Next, let $u_{\infty} \in H^{2}(0,1)$ be a unique solution of the limit equation (48), and

$$
q_{\infty}(x):=\frac{\nu^{1 / 2}}{u_{\infty}(x)}
$$

Let $w(x, t):=q(x, t)-q_{\infty}(x)$. Then

$$
\begin{aligned}
\mathcal{E}\left(q \mid q_{\infty}\right) & :=\mathcal{E}(q(t))-\mathcal{E}\left(q_{\infty}\right)=\frac{1}{2} \int_{0}^{1} w_{x}\left(q+q_{\min }\right)_{x} d x+\nu^{-\frac{1}{2}} \int_{0}^{1}\left(q f(x, t)-q_{\infty}(x) f_{\infty}(x)\right) d x \\
& =-\frac{1}{2} \int_{0}^{1} w\left(q+q_{\min }\right)_{x x} d x+\nu^{-\frac{1}{2}} \int_{0}^{1} w f_{\infty}(x) d x+\nu^{-\frac{1}{2}} \int_{0}^{1} q\left[f-f_{\infty}(x)\right] d x \\
& =-\frac{1}{2} \int_{0}^{1} w w_{x x} d x+\nu^{-\frac{1}{2}} \int_{0}^{1} q\left[f-f_{\infty}(x)\right] d x=\frac{1}{2} \int_{0}^{1} w_{x}^{2} d x+\nu^{-\frac{1}{2}} \int_{0}^{1} q\left[f-f_{\infty}(x)\right] d x
\end{aligned}
$$

From here we find that

$$
\begin{aligned}
\frac{d}{d t} \mathcal{E}\left(q \mid q_{\infty}\right) & =-\int_{0}^{1} w_{t}\left[w_{x x}-\nu^{-\frac{1}{2}}\left(f-f_{\infty}(x)\right)\right] d x+\nu^{-\frac{1}{2}} \int_{0}^{1} q f_{t} d x \\
& =-\int_{0}^{1} q^{2}\left[w_{x x}-\nu^{-\frac{1}{2}}\left(f-f_{\infty}(x)\right)\right]^{2} d x+\nu^{-\frac{1}{2}} \int_{0}^{1} q f_{t} d x
\end{aligned}
$$

i.e.

$$
\begin{gathered}
\frac{1}{2} \frac{d}{d t} \int_{0}^{1} w_{x}^{2} d x+\int_{0}^{1} q^{2}\left[w_{x x}-\nu^{-\frac{1}{2}}\left(f-f_{\infty}(x)\right)\right]^{2} d x= \\
-\nu^{-\frac{1}{2}} \int_{0}^{1} q_{t}\left[f-f_{\infty}(x)\right] d x \leqslant \frac{1}{2} \int_{0}^{1} q^{2}\left[w_{x x}-\nu^{-\frac{1}{2}}\left(f-f_{\infty}(x)\right)\right]^{2} d x+ \\
\frac{\nu^{-1}}{2} \int_{0}^{1} q^{2}\left[f-f_{\infty}(x)\right]^{2} d x
\end{gathered}
$$

whence

$$
\frac{d}{d t} \int_{0}^{1} w_{x}^{2} d x+\int_{0}^{1} q^{2}\left[w_{x x}-\nu^{-\frac{1}{2}}\left(f-f_{\infty}(x)\right)\right]^{2} d x \leqslant \nu^{-1} \int_{0}^{1} q^{2}\left|f-f_{\infty}(x)\right|^{2} d x .
$$

Let us denote by

$$
Z(x, t):=w_{x}-\nu^{-\frac{1}{2}} \int_{0}^{x}\left(f-f_{\infty}(s)\right) d s, \quad K(x, t):=\int_{0}^{x}\left(f-f_{\infty}(s)\right) d s .
$$

As $Z(0, t)=Z(1, t)=K(0, t)=K(1, t)=0$ for $t>0$, then by the Poincaré inequality and (46), one has

$$
\frac{\nu \pi^{2}}{A_{+}^{2}} \int_{0}^{1} Z^{2} d x \leqslant \int_{0}^{1} q^{2} Z_{x}^{2} d x, \quad \pi\|K(\cdot, t)\|_{2} \leqslant\left\|K_{x}(\cdot, t)\right\|_{2}=\left\|f(\cdot, t)-f_{\infty}\right\|_{2} .
$$


Taking into account

$$
\begin{aligned}
\int_{0}^{1} Z^{2} d x & =\int_{0}^{1} w_{x}^{2} d x-2 \nu^{-\frac{1}{2}} \int_{0}^{1} w_{x} K d x+\nu^{-1} \int_{0}^{1} K^{2} d x \\
& \geqslant \frac{1}{2} \int_{0}^{1} w_{x}^{2} d x-\nu^{-1} \int_{0}^{1} K^{2} d x
\end{aligned}
$$

and (65) we deduce that

$$
\int_{0}^{1} v^{2}\left[w_{x x}-\nu^{-\frac{1}{2}}\left(f-f_{\infty}(x)\right)\right]^{2} d x \geqslant \frac{\nu \pi^{2}}{A_{+}^{2}}\left[\frac{1}{2} \int_{0}^{1} w_{x}^{2} d x-\frac{1}{\pi^{2} \nu}\left\|f(., t)-f_{\infty}(x)\right\|_{2}^{2}\right] .
$$

Next, using again (46) one estimates

$$
\begin{aligned}
\nu^{-1} \int_{0}^{1} q^{2}\left|f-f_{\infty}(x)\right|^{2} d x+\frac{1}{A_{+}^{2}} \int_{0}^{1}\left\|f(., t)-f_{\infty}(x)\right\|_{2}^{2} \leqslant \\
\left(\frac{1}{A_{-}^{2}}+\frac{1}{A_{+}^{2}}\right)\left\|f(., t)-f_{\infty}(x)\right\|_{2}^{2}:=D(t) .
\end{aligned}
$$

So, by (64) one obtains

$$
\frac{d}{d t} \int_{0}^{1} w_{x}^{2} d x+\frac{\nu \pi^{2}}{2 A_{+}^{2}} \int_{0}^{1} w_{x}^{2} d x \leqslant D(t) .
$$

Consequently, it follows that

$$
\int_{0}^{1} w_{x}^{2} d x \leqslant e^{-B t}\left[\left\|w_{0, x}\right\|_{2}^{2}+\int_{0}^{t} D(s) e^{B s} d s\right]
$$

where $B$ is from (50). It remains only to show that the right-hand side of (67) tends to zero as $t \rightarrow \infty$. This is true immediately, if

$$
\int_{0}^{\infty} D(s) e^{B s} d s<\infty
$$

But if

$$
\lim _{t \rightarrow \infty} \int_{0}^{t} D(s) e^{B s} d s=+\infty
$$

then after an application of the L'Hopital's rule one has:

$$
\lim _{t \rightarrow \infty} e^{-B t} \int_{0}^{t} D(s) e^{B s} d s=\lim _{t \rightarrow \infty} \frac{D(t)}{B}=0 .
$$

Therefore, we conclude that (67) together with (62) and (51) implies asymptotic convergence estimate (49). 
Remark 3.2. One can check that results of Theorem 3.1 are consistent with those of Theorem 2.1. Indeed, in the time-homogeneous case $f(x, t)=f_{0}(x)$ formulae (44)-(45) and (46)-(47) coincide with (19) and (20), respectively. This is verified by the direct substitution of $N_{\infty}=0$ into the former formulae. Also the asymptotic convergence estimate (49)-(50) is consistent with (21) in this case.

Corollary 3.3. Conditions of Theorem 3.1 imply also the direct exponential asymptotic convergence of $u(\cdot, t)$ to $u_{\infty}$ in $H^{1}(0,1)$. Namely, there exists a constant $C_{1}$ depending on $\nu, f(x, t)$ and $u_{0}, f_{0}, f_{\infty}$ such that for all $t>0$ it holds

$\left\|u(\cdot, t)-u_{\infty}\right\|_{H^{1}} \leqslant C_{1} e^{-B t}\left[\left\|u_{0}-u_{\infty}\right\|_{H^{1}}+\int_{0}^{t}\left\|f(., t)-f_{\infty}\right\|_{2}^{2} e^{B s} d s\right] \rightarrow 0$ as $t \rightarrow \infty$

Proof. The statement follows from (49), uniform bounds (46) and the following pointwise bounds:

$$
\begin{aligned}
& \left(1+\frac{N_{\infty}+P_{0}}{\nu}\right)^{-1} \leqslant u_{\infty}(x) \leqslant\left(1-\frac{N_{\infty}+P_{0}}{\nu}\right)^{-1}, \\
& \left(1+R_{0}\right)^{-1} \leqslant u_{0}(x) \leqslant\left(1-R_{0}\right)^{-1} \text { for } x \in(0,1) .
\end{aligned}
$$

Note, that the pointwise bounds for $u_{\infty}$ follow from the estimate

$$
\left|u_{\infty}^{-1}(x)-1\right| \leqslant\left\|\left(u_{\infty}^{-1}\right)_{x}\right\|_{2} \leqslant \nu^{-1}\left\|\int_{0}^{x} f_{\infty}(s) d s\right\|_{2} \text { for all } x \in(0,1),
$$

and (42), (59). In (68) we also used that $R_{0}<1$ and $N_{\infty}+P_{0}<\nu$ hold by assumptions (44) $-(45)$.

Example 3.4. Here, we calculate an explicit form of the asymptotic convergence estimate (49)-(50) for the particular right-hand side

$$
f(x, t)=\min \{1,1 / t\} \cos (\pi x), \quad\|f(\cdot, t)\|_{2} \rightarrow 0 \text { as } t \rightarrow \infty ;
$$

and the initial data

$$
u_{0}=u_{\infty}=1
$$

In this case,

$$
\begin{aligned}
& R_{0}=0, \quad P_{0}=\left\|\int_{0}^{x} \cos (\pi s) d s\right\|_{2}=\frac{1}{\sqrt{2}}, \\
& N_{\infty}=\int_{1}^{\infty}\left\|\int_{0}^{x} \frac{\cos (\pi s)}{t^{2}} d s\right\|_{2} d t=\frac{1}{\sqrt{2}} .
\end{aligned}
$$

Therefore, conditions (44)-(45) reduce to

$$
\nu \in\left(\frac{3}{\sqrt{2}}+2, \infty\right)
$$


while constants (47) to

$$
A_{ \pm}=\frac{\nu}{\nu \mp[3 / \sqrt{2}+2]}
$$

Hence, constants in (50) become

$$
C=\frac{2}{\nu^{5 / 2}}\left(\nu^{2}+[3 / \sqrt{2}+2]^{2}\right), \quad B=\frac{\pi^{2}[\nu-3 / \sqrt{2}-2]^{2}}{2 \nu} .
$$

Finally, estimate (49) reduces in this example to

$$
\left\|u^{-1}(\cdot, t)-1\right\|_{H^{1}} \leqslant \frac{C}{2} e^{-B t}\left(\int_{0}^{1} e^{B s} d s+\int_{1}^{t} \frac{1}{s^{2}} e^{B s} d s\right) \text { for all } t>0 .
$$

For sufficiently large $t>1$ the last estimate implies that

$$
\left\|u^{-1}(\cdot, t)-1\right\|_{H^{1}} \leqslant \frac{C}{2} \exp \{-B t\}\left(\frac{2}{B t^{2}}+\frac{e^{B}-1}{B}\right) \text { as } t \rightarrow \infty
$$

with the exponential decay rate $B$ from (69)).

\section{Acknowledgements}

GK would like to acknowledge support from the Oxford Centre for Industrial and Applied Mathematics (OCIAM) of the University of Oxford. Part of this research was performed during participation of one of the authors at the thematic research program "Mathematical Biology" organised by the Mittag-Leffler Institute.

\section{References}

[1] M. A Fontelos, G. Kitavtsev, and R. M. Taranets. Asymptotic decay and non-rupture of viscous sheets. Z. Angew. Math. Phys., 69(3):79, 2018.

[2] M. L. Storm. Heat conduction in simple metals. J. Appl. Phys., 22(7):940-951, 1951.

[3] J. L. Vazquez. The porous medium equation. Mathematical theory. Oxford mathematical monographs, Clarendon Press, Oxford, 2007.

[4] G. Rosen. Nonlinear heat conduction in solid $H_{2}$. Phys. Rev. B, 19(4):2398-2399, 1979.

[5] G. Bluman and S. Kumei. On the remarkable nonlinear diffusion equation. J. Math. Phys., 21:1019-1023, 1980.

[6] J. R. King. Some non-local transformations between nonlinear diffusion equations. J. Phys. A: Math. Gen., 23:5441-5464, 1990. 
[7] A. Segatti G. Schimperna and S. Zelik. On a singular heat equation with dynamic boundary conditions. Asymptotic Analysis, 97:27-59, 2016.

[8] O. Penrose and P. C. Fife. Thermodynamically consistent models of phase-field type for the kinetics of phase transitions. Phys. D, 43:44-62, 1990.

[9] S. Zheng. Global existence for a thermodynamically consistent model of phase field type. Differential Integral Equations, 5(2):241-253, 1992.

[10] A. Oron, S. H. Davis, and S. G. Bankoff. Long-scale evolution of thin liquid films. Rev. Mod. Phys., 69(3):931-980, 1997.

[11] B. Perthame J. F. Gerbeau. Derivation of viscous Saint-Venant system for laminar shallow water; numerical validation. Discrete Contin. Dyn. Syst. Ser. B, 1(1):89-102, 2001.

[12] D. Bresch. Shallow-water equations and related topics. In C. M. Dafermos and M. Pokorny, editors, Handbook of differential equations. Evolutionary equations, volume 5, pages 1-105. Elsevier, 2009.

[13] D. Bresch and P. Noble. Mathematical derivation of viscous shallow-water equations with zero surface tension. Indiana Univ. Math. J., 60(4):1137-1170, 2011.

[14] J. Eggers and M. A. Fontelos. Singularities: Formation, Structure, and Propagation. Cambridge Texts in Applied Mathematics, Cambridge, 2015.

[15] J. A. Carrillo, A. Jüngel, P. A. Markowich, G. Toscani, and A. Unterreiter. Entropy dissipation methods for degenerate parabolic problems and generalized sobolev inequalities. Monatshefte für Mathematik, 133(1):1-82, 2001.

[16] J. L. Vazquez. Smoothing and decay estimates for nonlinear diffucion equations. Equations of porous medium type. Oxford Lecture Series in Mathematics and Applications, 33. Oxford University Press, Oxford, 2006.

[17] J. Dolbeault and G. Karch. Large time behaviour of solutions to nonhomogeneous diffusion equations. Banach Center Publications, 74:133, 2006.

[18] G. Kitavtsev, P. Laurencot, and B. Niethammer. Weak solutions to lubrication equations in the presence of strong slippage. Methods Appl. Anal., 18(2):183202, 2011.

[19] I. Bihari. A generalization of a lemma of Bellman and its application to uniqueness problems of differential equations. Acta Mathematica Hungarica, 7(1):81-94, 1956. 